\title{
BISTATIC SAR TOMOGRAPHY: PROCESSING AND EXPERIMENTAL RESULTS
}

\author{
Sergi Duque ${ }^{(1)}$, Paco López-Dekker ${ }^{(1)(2)}$, Juan C. Merlano ${ }^{(1)}$, and Jordi J. Mallorqui ${ }^{(1)}$ \\ ${ }^{(1)}$ Remote Sensing Laboratory (RSLab) - UPC, Spain \\ ${ }^{(2)}$ DLR, Germany
}

\begin{abstract}
This paper presents across-track tomography applied to a bistatic geometry with fixed receivers. This kind of geometry can overcome some of the classical monostatic tomography limitations such as temporal decorrelation and irregular baseline distribution. The Remote Sensing Laboratory (RSLab) of the Universitat Politècnica de Catalunya (UPC) has implemented a SAR Bistatic Receiver for INterferometric Applications, SABRINA, with 4-channels. SABRINA has been used to carry out a bistatic tomographic experiment. The acquired data has been processed with different tomographic methods and their performances compared.
\end{abstract} line

Index Terms - SAR, Biestatic, Tomography, Multibase-

\section{INTRODUCTION}

During the last decades, Synthetic Aperture Radar (SAR) has become an important tool for Earth Observation. Of the many techniques that have arisen using SAR, one of the most popular is SAR interferometry (InSAR). InSAR is a well-known technique for measuring topography. It consists of using two different SAR images acquired from slightly different looking angles. For each resolution cell of the SAR image, InSAR retrieves the height of the mean or dominant scattering center. Furthermore, with only two images it is impossible to determine the heights of the different scattering centers located within a resolution cell. Scattering at different heights within the same resolution cell can be due to the penetration of the radiation through different scattering layers, due to the presence or different scattering sources or because the topography is steep enough to generate foreshortening or layover. Urban areas are a clear example of the latter case.

Multibaseline (MB) techniques have appeared as a natural evolution of InSAR. They are based on using more than two images of the scene. Applying tomographic techniques to a MB data set, it is possible to retrieve for each resolution cell the distribution of backscattered power as function of height

This work has been supported by the Spanish MCYT funds under the projects TEC2008-06764-C02-01 and TEC2007-65690/TCM, the Spanish Ramon y Cajal program, the Catalan Commission for Research (CIRIT) and the Spanish MEC granting the first author (AP2005-1484). above ground. MB techniques have been studied using monostatic spaceborne [1] and airborne [2] sensors. For these sensors, MB techniques require several passes to obtain the data needed to complete the baseline set. The resulting baselines depend on the transmitter's path, which is difficult to control accurately. As a result, the baseline sampling is irregular and advanced spectral estimation techniques are needed to obtain the height profile. Also, the scene might have changed between the different passes, introducing temporal decorrelation and therefore MB focusing may fail.

If multiple fixed-receivers under a bistatic approach were placed observing the region of interest, forming a set of baselines, some problems related to the classical monostatic tomography could be overcome. For example, using a single transmitter and several receivers, all data could be taken in a single-pass configuration and thus eliminating the temporal decorrelation.

The Remote Sensing Laboratory (RSLab) of the Universitat Politècnica de Catalunya (UPC) is investigating bistatic configurations that use ground-based fixed receivers and orbital sensors as transmitters of opportunity. In this context, a first C-band receiver named SABRINA (SAR Bistatic Receiver for INterferometric Applications) with 4-channels [3] has been implemented and successfully tested using ESA's ERS-2 and ENVISAT satellites as transmitters of opportunity. Also, a X-band version for TerraSAR-X is available. SABRINA is usually placed at locations with a good view of the scene, like the top of a hill or the roof of a tall building.

This paper presents across-track tomography applied to a bistatic geometry with fixed receivers. The next section shows a scheme of the acquisition geometry and discusses some classical inversion methods applied to the bistatic case. Section 3 presents an experiment that has been carried out in order to show the performance of the bistatic tomography over real data with ground truth. Finally the conclusions of this work are described in Section 4.

\section{THEORY}

The geometry considered in this study is illustrated in Figure 1. A satellite is used as a transmitter of opportunity, while a set of $\mathrm{N}$ receivers are placed at a height above the ground, $H_{R x}$. Its vertical separation allows to form a set of baselines 


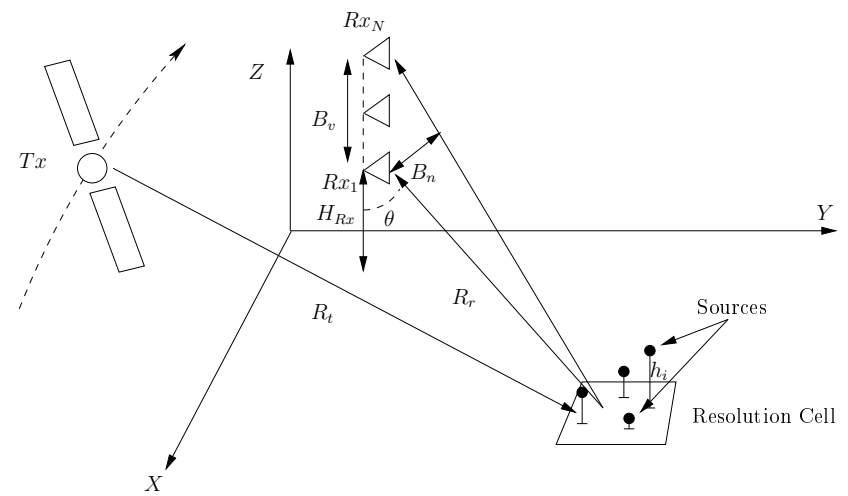

Fig. 1. Bistatic tomographic geometry. The figure shows a satellite used as a transmitter of opportunity and $\mathrm{N}$ fixed receivers forming a set of vertical baselines.

separated $\Delta B v$. Each resolution cell of the scene can contain several scattering sources located at different heights, $h_{i} . R_{t}$ and $R_{r}$ represent the range of a resolution cell to the satellite and to the receivers respectively, $\theta$ is the incidence angle for a specific resolution cell and $B v_{n}$ and $B n_{n}$ are the nth vertical and normal baselines.

\subsection{Tomographic processing techniques}

The complex values corresponding to a particular ground range cell acquired at the $\mathrm{N}$ receivers taking into account the multiplicative speckle noise can be expressed as the following vector

$$
y=\sum_{i=1}^{N_{s}} \sqrt{\tau_{i}} x_{i} \odot a\left(\omega^{i}\right)+v
$$

where $\odot$ is the Hadamard product, $N_{s}$ is the number of sources, $\tau_{i}$ is the reflectivity for the i-th source, $y$, is the $\mathrm{N}$-dimensional vector for the acquired data, $x_{i}$ for the multiplicative noise, $a\left(\omega_{i}\right)$ the steering vector at frequency $\omega^{i}$ associated to a target height of $h_{i}$ and $v$ is the thermal noise vector. This expression is valid for a fixed receiver bistatic system with $N$ receivers just taking into account that the steering vector for a target at height $h_{i}$ is

$$
a\left(\omega^{i}\right)=\left[1, e^{j \omega_{1}^{i}}, e^{j \omega_{2}^{i}}, \ldots, e^{j(N-1) \omega_{N-1}^{i}}\right]
$$

being

$$
\omega_{k}^{i}=\frac{2 \pi}{\lambda} \cdot \frac{B n_{k}}{R_{r} \cdot \sin (\theta)} \cdot h_{i} .
$$

Notice that the factor $2 \pi$ is due to the one-way bistatic path while $R_{r}$ is the distance from target to receiver.

The goal of the inversion methods is to obtain the heights of the targets in the same resolution cells $\left(h_{i}\right)$. The different methods can be classified as non-parametric and parametric. The non-paremetric methods employed in this work are
Beamforming and Capon while the parametric used has been the MUltiple SIgnal Classification (MUSIC).

The nominal height resolution, taking the beamforming as a reference, for the bistatic geometry with fixed receivers is given by

$$
\Delta h=\frac{\lambda R_{r}}{B v},
$$

being $\lambda$ the wavelength and $B v$ the total vertical baseline. Also, the maximum height range without ambiguity goes from $-h_{\max }$ up to $h_{\max }$, being $h_{\max }$

$$
h_{\max }=\frac{\lambda R_{r}}{2 \Delta B v}
$$

where $\Delta B v$ is the vertical equidistant separation between receivers.

\subsection{Non-Parametric methods}

The only a priori assumption made by this methods is the Born approximation.

\subsubsection{Traditional Beamforming}

Beamforming (BF) was the first proposed method to solve the inversion problem for MB InSAR acquisitions. This approach can be seen as a Finite Impulse Response (FIR) filter. This filter finds the coherent combination of all the acquisitions. Taking into account the steering vector and the data $y$, the output of the filter is

$$
\hat{x}_{B F}(\omega)=\frac{1}{N} a^{H}(\omega) \cdot y .
$$

The performance of BF is poor in terms of resolution and it presents a low Peak SideLobe Ratio (PSLR).

\subsubsection{Capon}

Capon is another non-parametric method that can be used as frequency estimator. This approach constructs a bank of adaptative band-pass FIR filters of length N. These filters are designed so that the power of the filtered signal is minimized with the constrain that, in the frequency domain, the filter must have unity gain at the selected frequency. For a baseline dataset with a covariance matrix R, the optimal filter for frequency $\omega$ is given by the solution to

$$
\min h^{H} \cdot R \cdot h, \text { subject to } h^{H} \cdot a=1
$$

If there are a small number of dominant frequency components, which implies a small number of bright targets, the filters tend to place nulls at the rest of the frequencies. However, the filters present a large frequency response, side lobes, at those frequencies where there is interfering signal. The resolution improvement is due to the ability of placing nulls at 
the dominant frequencies of the filters. It has to be said that if the number of dominant frequencies to be suppressed increases, the ability of the algorithm to place nulls decreases. In an extreme case, there is no way to estimate the number of sources if it is equal or larger than the number of acquisitions $\left(N_{s} \geq N\right)$.

The output of the Capon filter is

$$
\hat{x}_{\text {Capon }}(\omega)=\frac{a^{H}(\omega) \hat{R}^{-1} y}{a^{H}(\omega) \hat{R}^{-1} a(\omega)},
$$

where $\hat{R}$ is the estimated covariance matrix, which needs a number of looks greater than the number of receivers $\left(L_{r g-a z}>N\right)$, as

$$
\hat{R}=\frac{1}{L_{r g-a z}} \sum_{l=1}^{L} y(l) y^{H}(l) .
$$

Its inverse will exist if the noise term has a positive defined covariance matrix and the number of looks is larger or equal than the number of acquisitions $L_{r g-a z} \geq N$.

\subsection{Parametric methods}

\subsubsection{MUSIC}

The assumption made by MUSIC, apart from the Born approximation, is that the number of sources, $N_{s}$, is a priori known. MUSIC is a subspace-based algorithms wich supposes that the noise and the signal are uncorrelated. MUSIC decomposes the covariance matrix in its eigenvalues and eigenvectors. For this algorithm, the covariance matrix is computed as in the classical Capon method. The decomposition of the estimated covariance matrix gives the eigenvalues $\hat{\lambda}_{1} \geq \hat{\lambda}_{2} \geq \ldots \geq \hat{\lambda}_{N}$, accordingly to the number of sources imposed the greater eigenvalues are associated to the signal subspace and the lower to the noise subspace. Thus corresponding eigenvectors related to the signal subspace are $\hat{f}_{1}, \ldots, \hat{f}_{N} s$ while to the noise subspace are $\hat{g}_{1}, \ldots, \hat{g}_{N-N s}$. The $\hat{f}_{i}$ vectors form the $N \times N_{s}$ element matrix $\hat{F}=\left[\hat{f}_{1}, \ldots, \hat{f}_{N_{s}}\right]$ and the $\hat{g}_{i}$ form the $N \times N-N_{s}$ matrix $\hat{G}=\left[\hat{g}_{1}, \ldots, \hat{g}_{N-N_{s}}\right]$. The pseudo-reflectivity can be expressed by

$$
\hat{P}_{M U S I C}(\omega)=\frac{1}{a^{H}(\omega) \hat{G} \hat{G}^{H} a(\omega)} .
$$

The subspace of the noise eigenvectors has to be at least of dimension one, which means $N>N_{s}$. This method presents a good resolution when the number of assumed sources is the correct one, but it does not performs well if it is not correct.

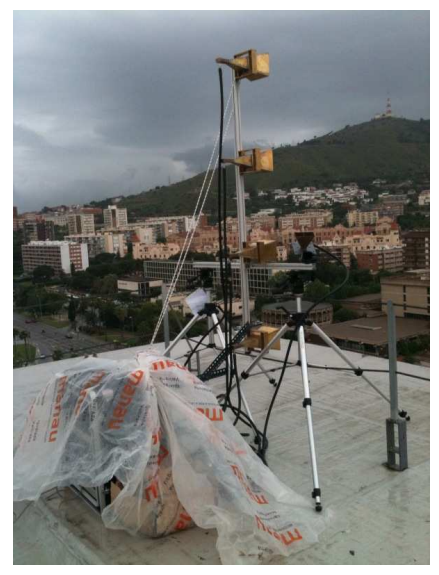

Fig. 2. SABRINA-C on top of a building. The antennas are distributed vertically with a separation of $62 \mathrm{~cm}$, being the longest baseline $1.86 \mathrm{~m}$.

\section{RESULTS}

\subsection{Experimental set-up}

An experiment with a bistatic geometry with fixed receivers has been carried out in order to apply the inversion methods explained before and get the height of different sources inside a resolution cell. The satellite ENVISAT was used as transmitter of opportunity and the bistatic receiver, SABRINA-C, was deployed on top of a $54 \mathrm{~m}$ tall building, as illustrated in Figure 2. The antennas corresponding to each available channel were vertically distributed with a separation of $62 \mathrm{~cm}$, being the longest baseline $1.86 \mathrm{~m}$. Three Polarimetric Active Radar Calibrators (PARC's) were used as a control points to validate the results. These PARCs consist in an antenna receiving the direct signal from the satellite, an amplification chain and a second antenna redirecting the signal towards the receiver. The first two PARCs were located at a $16.8 \mathrm{~m}$ tall building, one at ground level and the other one on the roof. The vertical separation is small enough to assume that both scatters will be within the same resolution cell. Figure 3 shows the building and the deployed PARCs. As the two PARCs can be considered identical, they should be seen with the same intensity once the inversion methods are applied.

Taking into account that the receiver-PARCs range is about $442 \mathrm{~m}$, the nominal resolution in height is 13.4 ( $\Delta h=$ $13.4 \mathrm{~m})$ and the height of ambiguity $20 \mathrm{~m}\left(h_{\max }=20 \mathrm{~m}\right)$. Thus, it would be possible distinguish the two targets. The third control point was placed on top of another building, being the main scatterer in its resolution cell.

\subsection{Tomographic results}

The tomographic processing is applied over the different interferometric phases once the known topography has been removed. This known topography is removed using a low 


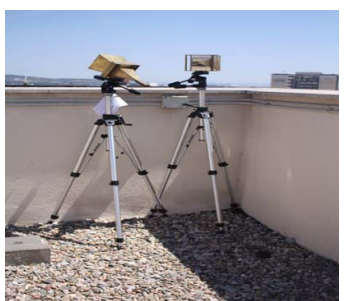

(a)

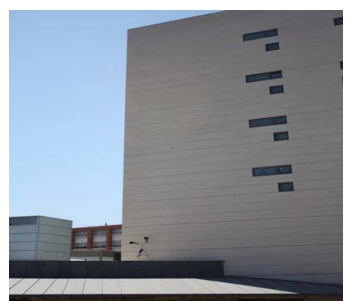

(b)
Fig. 3. Control points employed on the same resolution cell. One on the top 3(a) and at ground level 3(b) of the building.

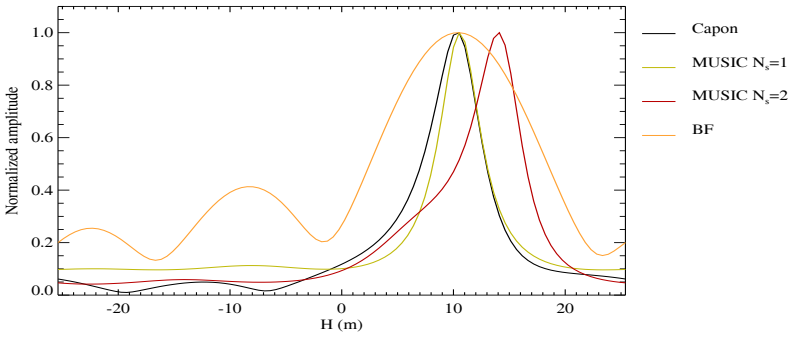

Fig. 4. Graph of the different inversion methods employed over a resolution cell with only a single target above the ground. Capon is represented in black, Beamforming in orange, MUSIC assuming only one and two targets are in beige and red, respectively.

resolution DEM from the Shuttle Radar Topography Mission (SRTM) has been employed. The inversion methods applied over the resolution cell with a single scatterer are showed in Figure 4. The graph represents the normalized amplitude distribution among the possible heights without ambiguity for the different methods. Capon is represented in black, Beamforming in orange, MUSIC assuming only one $\left(N_{s}=1\right)$ and two $\left(N_{s}=2\right)$ targets are in beige and red, respectively.

All methods, except MUSIC assuming two targets $\left(N_{s}=\right.$ 2 ), find only one target located at $10 \mathrm{~m}$ above the ground, which is a reasonable height for the building. Beamforming shows a wider mainlobe and presents a low PSLR, as it was expected. Capon and MUSIC assuming one target performt well. However, MUSIC assuming two targets is no so accurate as it can be seen in the graph.

Figure 5 shows the result of the methods applied to the resolution cell with two targets. All methods, except MUSIC with $N_{s}=1$, are able to find the contribution of the two targets. Also, all of them place the first source at $0 \mathrm{~m}$ and the second one around $12 \mathrm{~m}, 4.8 \mathrm{~m}$ less than the real value. This misplacement of the second target can be caused by an error in the estimation of the geometric parameters, such as the incidence angle or the range to the receiver. Beamforming shows the widest lobes and a poor PSLR. Capon is the only method that clearly shows two main targets with the same intensity. Figure 6 shows the targets found by the different

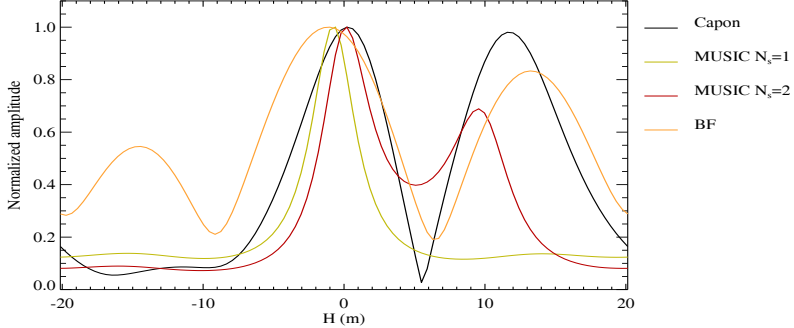

Fig. 5. Graph of the inversion methods employed over a resolution cell with two dominant targets.

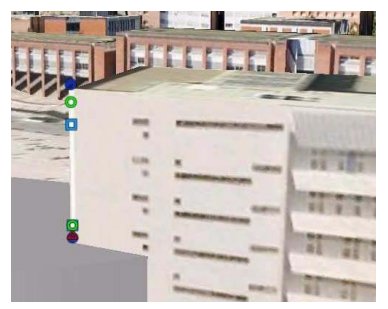

Fig. 6. Targets found by the different methods geocoded. Capon in green rings, BF in blue circles, MUSIC with $N_{s}=1$ in red triangle and MUSIC with $N_{s}=2$ in blue squares.

methods geocoded.

\section{CONCLUSIONS}

The results obtained from the bistatic tomographic experiment validate the bistatic tomography as a powerful technique for $3 \mathrm{D}$ imaging. This kind of geometry is able to acquire tomographic data with only one satellite pass, avoiding the temporal decorrelation, and with a uniform distribution of the baselines. As a result of the inversion method comparison, the BF shows the wider lobes and low PSLR, MUSIC only presents a good performance if the number of sources is the correct one and Capon has a high PSLR and a good trade-off between the peak width and the accuracy of the peaks position.

\section{REFERENCES}

[1] G. et al Fornaro. Three-dimensional multipass SAR focusing: experiments with long-term spaceborne data. IEEE TGRS, 43:702-714, April 2005.

[2] A. Reigber and A. Moreira. First demonstration of airborne SAR tomography using multibaseline L-band data. IEEE TGRS, 38:2142-2152, September 2000.

[3] J. Sanz-Marcos, P. Lopez-Dekker, J. J. Mallorqui, A. Aguasca, and P. Prats. SABRINA: a SAR bistatic receiver for interferometric applications. IEEE Geoscience and Remote Sensing Letters, Vol 4:pages 307-311, 2007. 